\title{
Study on the Microwave Permittivity of Single-walled Carbon Nanotube
}

\author{
Xiaolai Liu \\ Department of Physics and Electronic Science \\ Beijing University of Chemical Technology \\ Beijing 100029, China
}

Donglin Zhao

Education Ministry Key Laboratory of Science and Technology of Controllable Reactions

Institute of Carbon Fiber and Composites

Beijing 100029, China

The research is financed by the Science and Technology Nova Program of Beijing of China (No.H020821280120) and the Science Foundation for Post-doctor of China (No.2001030521). (Sponsoring information)

\begin{abstract}
In this article, we studied the microwave permittivity of the complex of the single-walled carbon nanotube and paraffin in $2-18 \mathrm{GHz}$. In the range, the dielectric loss of single-walled carbon nanotube is higher, and the real part and the imaginary part of the dielectric constant decrease with the increase of frequency, and the dielectric constant possesses the property of dispersion frequency response. The $\varepsilon^{\prime}, \varepsilon^{\prime \prime}$ and tan $\delta$ of the complex of the single-walled carbon nanotube and paraffin increase with the increase of the content of carbon tubes, and it presents good second-order function relationship with the volume fraction of carbon in certain range. The polarization dispersion dielectric response is the main reason that the single-walled nanotube absorbs and wastes microwaves.
\end{abstract}

Keywords: Single-walled carbon nanotube, Microwave permittivity, Microstructure, Microwave absorption

\section{Introduction}

Since Iijima found the carbon nanotube in 1991 (S. lijima, 1991, P.56), the carbon nanotube has been broad concerned by people, and it has been the research hotspot in many scientific domains such as chemistry, physics, and material science. There are many methods such as graphite electric arc method (DC electric arc method), catalytic crack method, laser evaporation graphite rod method, pyrolytic polymer method, flame method, ion (electron beam) radiation method, electrolysis method and model carbonization method to be used to prepare nanotube (G, Ya, Slepyan, 2001, P.121-123, Bachtold A, 2001, P.1317, Tans SJ, 1998, P.49, Wagner HD, 1998, P.188, Planeix JM, 2001, P.1447, Cheng HM, 2001, P.1317, Service RF, 1998, P.940, John W, 2003), and the method which takes Fe, Co, Ni and other metals as activators and catalyze and crack the hydrocarbon to prepare the carbon nanotube makes the industrialization production of carbon nanotube possible. Various forms and structures of carbon nanotube make the nanotube possess many potential applied values, for example, it can be used in the strengthening of materials, one-dimensional quanta lead, semiconductor materials, activator carrier, molecule absorbent, tunnel scanning and the detector of the atom force microscope. The carbon nanotube has many characters such as small tube diameter and big length-diameter ratio, and the diameter is in tens nanometers, and the axial length of the tube is on the level from micron to centimeter, and it is the thinnest fiber material, and this sort of special structure makes the carbon nanometer possess excellent mechanical performance and special electric performance, and the experiment shows that the Yong modulus of single multiple layers nanotube averagely is $1.8 \mathrm{TPa}$, and the curve intensity can achieve 14.2GPa (J, A, Roberts, 2004, P.4352-4356), and because the carbon nanotube is the one-dimensional material with hollow structure, so we can utilize the capillary phenomenon of carbon nanotube to fill some elements into the interior of the carbon nanotube and make the one-dimensional quanta line with special performance (K, G, Ong, 2002, P.82, C, Bower, 2002, P.3820, Zhao D L, 2001, P.2471). In a word, the preparation and application of carbon nanotube have been implemented for a long time, and in recent years, the research about the complex material of carbon nanotube has been one hotspot of the carbon nanotube appplicaiton, and there are few researches about the microwave permittivity of carbon nanotube, especial for the single-walled carbon nanotube and the function relationship between the effective dielectric constant of single-walled carbon nanotube and the volume 
fraction of carbon nanotube. In this article, we study the change rule of the dielectric constant of single walled carbon nanotube and paraffin complex with the volume fraction and frequency of carbon nanotube in $2-18 \mathrm{GHz}$, which will offer references for the application of single-walled carbon nanotube in the absorbing materials.

\section{Experiment}

The single-walled carbon nanotube used in the experiment is made by Shenzhen Nanotech Port Co., Ltd, and the diameter of the single-walled carbon nanotube is less than $2 \mathrm{~nm}$, and length is in $5-15 \mu \mathrm{m}$, and the specific surface area is $450-600 \mathrm{~m}^{2} / \mathrm{g}$. We use the coaxial line method to measure the dielectric constant of the single-walled carbon nanotube in the frequency range of $2-18 \mathrm{GHz}$, and the type of the network analyzer is HP8722ES. The preparation process of the dielectric constant testing sample of single-walled carbon nanotube is that putting the single-walled carbon nanotubes dispersed in the melting paraffin evenly, and casting the liquid compound of paraffin and single-walled carbon nanotubes into the copper loop standard flange, and testing the dielectric constants of the complex with the flange after solidification to eliminate the testing error induced by the gap between sample and flange. And the contents of single-walled carbon nanotube respectively are $5 \mathrm{wt} \%, 8 \mathrm{wt} \%, 10 \mathrm{wt} \%, 15 \mathrm{wt} \%$ and $20 \mathrm{wt} \%$.

\section{Results and discussions}

The function between the microwave and the agglomeration matter can be described by the complex dielectric constant $\varepsilon^{*}\left(\varepsilon^{*}=\varepsilon^{\prime}-i \varepsilon^{\prime \prime}, \varepsilon^{\prime}\right.$ is the real part of the dielectric constant, and $\varepsilon^{\prime \prime}$ is the imaginary part) and the complex electrical conductivity $\sigma^{*}$. And the relationship between the real part $\sigma^{\prime}(\omega)$ of the electrical conductivity and the imaginary part $\varepsilon^{\prime \prime}(\omega)$ of the complex dielectric constant is $\sigma^{\prime}(\omega)=\omega \varepsilon^{\prime \prime}(\omega)$, where, $\omega$ is the angle frequency of the electromagnetic wave (K, G, Ong, 2002, P.82, C, Bower, 2002, P.3820, Zhao D L, 2001, P.2471, A, K, Jonscher, 1983, P.138, P, Debye, 1945, P.257, E, Mouchon, 1996, P.323, Liu, Xiaolai, 2005, P.721, A, K, Jonscher, 1983, P.13). Most researchers mix the measured samples with the paraffin to test the electromagnetic parameters of the complex (Zhao D L, 2001, P.2471, A, K, Jonscher, 1983, P.138, P, Debye, 1945, P.257, E, Mouchon, 1996, P.323).

Because the specific surface area of the single-walled carbon nanotube is very large, and it is hard to be compacted, so we can not exactly measure the microwave dielectric constant of the pure single-walled carbon nanotube. In this article, we studied the change rule of the dielectric character of single-walled carbon nanotube and the paraffin with the content of the carbon nanotube, and researched the dielectric character of the samples with five different contents $(5 \mathrm{wt} \%, 8 \mathrm{wt} \%$, $10 \mathrm{wt} \%, 15 \mathrm{wt} \%$ and $20 \mathrm{wt} \%)$ in $2 \sim 18 \mathrm{GHz}$, and the real part and imaginary part of the dielectric constant of the paraffin used in the experiment were constants, i.e. $\varepsilon^{\prime}=2.26$ and $\varepsilon^{\prime \prime}=0$. Figure 1, Figure 2 and Figure 3 respectively show the change curves of the dielectric constants of the complex with different single-walled carbon nanotube and paraffin $\left(\varepsilon^{\prime}\right.$ and $\left.\varepsilon^{\prime \prime}\right)$ and the dielectric waste angle tangent $\left(\operatorname{tg} \delta=\varepsilon^{\prime \prime} / \varepsilon^{\prime}\right)$ with the frequency. We can see that with the increase of the content of single-walled carbon nanotube, the $\varepsilon^{\prime}, \varepsilon^{\prime \prime}$ and tg $\delta$ of the complex gradually increase, and with the increase of the frequency, $\varepsilon^{\prime}$ and $\varepsilon^{\prime \prime}$ gradually decrease, and it possesses obvious frequency response property, which is very beneficial to enhance the absorbing performance of absorbing materials in the wide frequency range.

The research about the complex electromagnetic property is an old and active topic (Liu, 2005, P.721, A, K, Jonscher, 1983, P.13, J, C, M, Gamett, 1904, P.385, J, C, M, Garnett, 1904, P.237, A, H, Sihvola, 1998, P.420, K, Bober, 1997 , P.101, G, F, Dionne, 1976, P.1708), and J.C. Maxwell Garnett educed the famous effective medium formula which was used to solve the complex dielectric constant in 1904, i.e. the Maxwell Garnett formula (A, K, Jonscher, 1983, P.13, J, C, M, Gamett, 1904, P.385). With the development of science and technology, many microwave apparatus, especially for the microwave absorbing materials developing from stealth technology, all need the materials with special electromagnetic property, but single material could not fulfill these special requirements, so we need prepare complex materials to implement these objectives, and though researches try to look for a sort of mathematical theoretical model to compute the microwave electromagnetic parameters of complex materials all along, but they could not establish a sort of general theoretical model (G, F, Dionne, 1976, P.1708).

The effective dielectric function of complex material is generally obtained by experiment and test or the establishment of proper mathematical model, and the later has important function in the material design, but up to now, there is not very satisfactory model, so researchers still try to find the optimal model all along. Bober et al (G, F, Dionne, 1976, P.1708) used Maxwell Garnett formula to respectively compute the complex dielectric constant and complex permeability for the complex materials which are respectively composed by resin with nickel zinc ferrite, mangan zinc ferrite, $\mathrm{Sr}$ ferric oxide and graphite in $10 \mathrm{GHz}$, and they found the Maxwell Garnett effective medium formula could approximately compute $\varepsilon^{\prime}$ for the complex material of nickel zinc ferrite and resin, but obvious warp would occur in $\varepsilon^{\prime \prime}$, $\mu^{\prime}$ and $\mu^{\prime \prime}$, and $\varepsilon^{\prime}, \varepsilon^{\prime \prime}, \mu^{\prime}$ and $\mu^{\prime \prime}$ all had very large warps in other sorts of complex material. Boner's study result also indicated that the dielectric constant $\left(\varepsilon^{\prime}\right.$ and $\left.\varepsilon^{\prime \prime}\right)$ and permeability $\left(\mu^{\prime}\right.$ and $\left.\mu^{\prime \prime}\right)$ of the complex materials which are respectively composed by resin with nickel zinc ferrite, mangan zinc ferrite, $\mathrm{Sr}$ ferric oxide and graphite and the volume fractions of ferrite and graphite accorded with the second-order multinomial relationship $\left(\varepsilon^{\prime}, \varepsilon^{\prime \prime}=\mathrm{Av}^{2}+\mathrm{Bv}+\mathrm{C}\right)$. Dionne et al studied the dielectric constants of the complex of rutile $\left(\varepsilon^{\prime}=100\right)$, anatase $\left(\varepsilon^{\prime}=48\right)$ and paraffin, and found that the warp would occur between measured value with computation value of Maxwell Garnett formula. Above research results 
indicate the Maxwell Garnett effective medium formula is not fit to compute the dielectric constant and permeability of the microwave complex materials.

From above discussions, we can see that we should look for other methods to compute the dielectric constant of the complex materials composed by single-walled carbon nanotube with different materials in the frequency band of microwave. Our research indicated that the dielectric constant measured value of the complex materials with different single-walled carbon nanotube contents had very good fitting relationship with the volume fraction of nanometer powder, and $\varepsilon^{\prime}$ and $\varepsilon^{\prime \prime}$ with the volume fraction of nanometer powder accord with the second-order multinomial relationship $\left(\varepsilon^{\prime}, \varepsilon^{\prime \prime}=\mathrm{Av}^{2}+\mathrm{Bv}+\mathrm{C}\right)$. Figure 4 and Figure 5 respectively are the second-order function curves of $\varepsilon^{\prime}$ and $\varepsilon^{\prime \prime}$ which are obtained by the fitting of dielectric constant measured value in $10 \mathrm{GHz}$ for the complex of single-walled carbon nanotube with paraffin with the volume fraction of nanometer powder (v). From the figures, we can see that $\varepsilon^{\prime}=-39.444 v^{2}+214.531 v-7.717, r^{2}=0.9999, \varepsilon^{\prime \prime}=2049.436 v^{2}-283.591 v+11.011, r^{2}=0.9929$, and they possess very good fitting relationship.

The researches also indicate that when the content of single-walled carbon nanotube is in $5 \mathrm{wt} \%-20 \mathrm{wt} \%$, and in the frequency range from $2 \mathrm{GHz}$ to $18 \mathrm{GHz}$, for different frequency testing sites, good second-order function relationship among $\varepsilon^{\prime}, \varepsilon^{\prime \prime}$ and $\mathrm{v}$, and the coefficients $\mathrm{A}, \mathrm{B}$ and $\mathrm{C}$ are different with the change of frequency. This research result is very important for the optimal design utilizing single-walled carbon nanotube and its polymer complex for the single narrow frequency, multiple frequencies and wide frequency absorbing wave.

In the single-walled carbon nanotube gives priority to the $\mathrm{sp}^{2}$ hybridization structure with carbon atom hexagonal close array, and the hybridization orbit presents electric moment, and the $\pi$ electron bond energy is small, and its combination force with atom is weak, and the effective quality is small and it presents high speed move and super polarization property, and in the alternation electric field function of microwave, the turning polarization of electric moment forms, and with the increase of frequency, the turning polarization of electric moment gradually trails the change of the exterior field, so this sort of polarization will decrease with the increase of frequency. In the turning process of electric moment, the energy loss will occur because of the non-elasticity mutual function with surrounding particles, and the move will trail the electric field, so the polarization dispersion dielectric response is the main reason that the single-walled nanotube absorbs and wastes microwaves.

\section{Conclusions}

(1) The dielectric loss of single-walled carbon nanotube is higher in the microwave frequency range of 2-18GHz, and the real part and the imaginary part of the dielectric constant will decrease with the increase of the frequency, and the dielectric loss angle tangent increases with the increase of frequency, and it possesses the property of polarization dispersion dielectric response.

(2) In the single-walled carbon nanotube gives priority to the $\mathrm{sp}^{2}$ hybridization structure with carbon atom hexagonal close array, and the hybridization orbit presents electric moment, and the $\pi$ electron bond energy is small, and its combination force with atom is weak, and the effective quality is small and it presents high speed move and super polarization property, and in the alternation electric field function of microwave, the turning polarization of electric moment forms, and the polarization dispersion dielectric response is the main reason that the single-walled nanotube absorbs and wastes microwaves.

(3) In the microwave frequency range of $2-18 \mathrm{GHz}$, the $\varepsilon^{\prime}$ and $\varepsilon^{\prime \prime}$ of the complex of single-walled carbon nanotube with paraffin have significant independence relationship with the content of single-walled carbon nanotube, and in the $5 \%-15 \%$ of the single-walled carbon nanotube content, the $\varepsilon^{\prime}$ and $\varepsilon^{\prime \prime}$ have good second-order function fitting relationship with the volume fraction of single-walled carbon nanotube, i.e. $\varepsilon^{\prime}, \varepsilon^{\prime \prime}=\mathrm{Av}{ }^{2}+\mathrm{Bv}+\mathrm{C}$.

\section{References}

A, H, Sihvola \& J, A, Kong. (1998). IEEE Trans, Geosc, Remote Sensing. No.26. P.420.

A, K, Jonscher. (1983). Dielectric Relaxation in Solids. (Chelsea Dielectric Press, London). P.13.

A, K, Jonscher. (1983). Dielectric Relaxation in Solids (Chelsea Dielectric Press, London). P.138.

Bachtold A, Hadley P \& Nakanishi T. (2001). Science. No.294. P.1317.

C, Bower, W, Zhu, D, Shalom, D, Lopez, P, L, Gammel, and S,Jin. (2002). Appl, PhysLett, 80, 3820.

Cheng HM, Yand QH \& Liu C. (2001). Carbon. No.294. P.1317.

E, Mouchon \& Ph, Colomban. (1996). J, Mater, Sci. No.31. P.323.

G, F, Dionne, J, F,F itzgerald, R, C, Aucoin. (1976). J, Appl, Phys. No.47. P.1708.

G,Ya, Slepyan, S, A, Maksimenko. (2001). Synthetic Metals. No.124. P.121-123.

J, A, Roberts, T, lmholt, Z, Ye. (2004). Journal of Applied Physics. No.95. P.4352-4356. 
J, C, M, Garnett. (1904). Philosoph, Trans, Roy, Soc, London. No.203. P.237.

J, C, M, Gamett. (1904). Philosoph, Trans, Roy, Soc, London. No.203. P.385.

John W, Schultz, Rick L, Moore. (2003). Mat, Res, Soc, Symp, Proc, 739 Materials Research Society.

K, Bober, R, H, Giles, J, Waldman. (1997). Inter, J, Infr, Mill,Wav. No.18. P.101.

K, G, Ong, K, Zheng, and C, Grimes. (2002). IEEE Sensors. J,2,82.

Liu Xiaolai. (2005). Microwave Permittivity of Multi-walled Carbon Nanotube AICAM. (in Chinese). P.721.

P, Debye. (1945). Polar Molecules (Dover, New York). P.257.

Planeix JM, Coustel N \& COQ B. (2001). J Am Carbon. No.39. P.1447.

Service RF. (1998). Science. No.281. P.940.

S, lijima. (1991). Nature (London). No.354. P.56.

Tans SJ, Verschueren AR \& Dekker C. (1998). Nature. No.393. P.49.

Wagner HD, Lourie O \& Feldman Y. (1998). Appl Phys Lett. No.72. P.188.

Zhao D L, Zhou W C, Wan W. (2001). Acta Phys, Sin. No.50. P.2471. Acta Physica Sinica. No.50. P.2471.

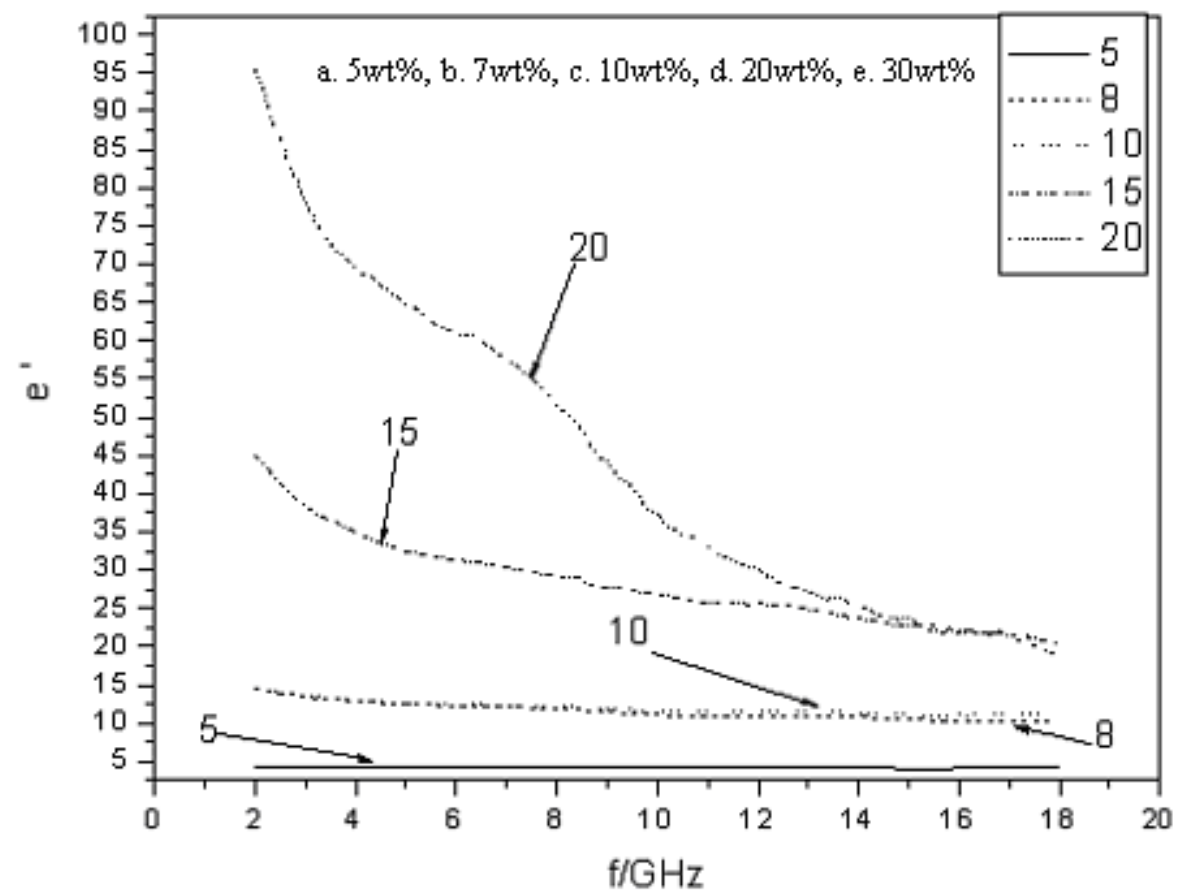

Figure 1. Relationship between $\varepsilon^{\prime}$ and frequency 


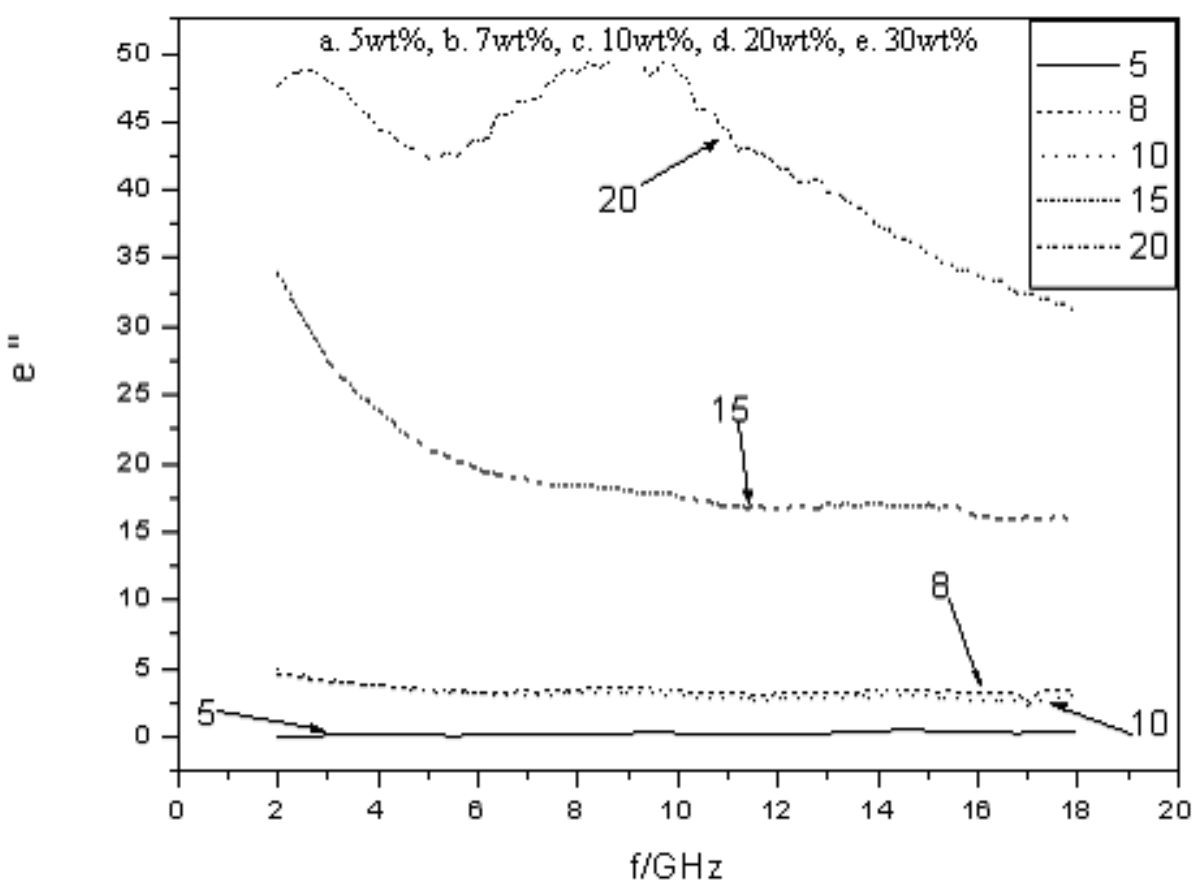

Figure 2. Relationship between $\varepsilon^{\prime \prime}$ and frequency

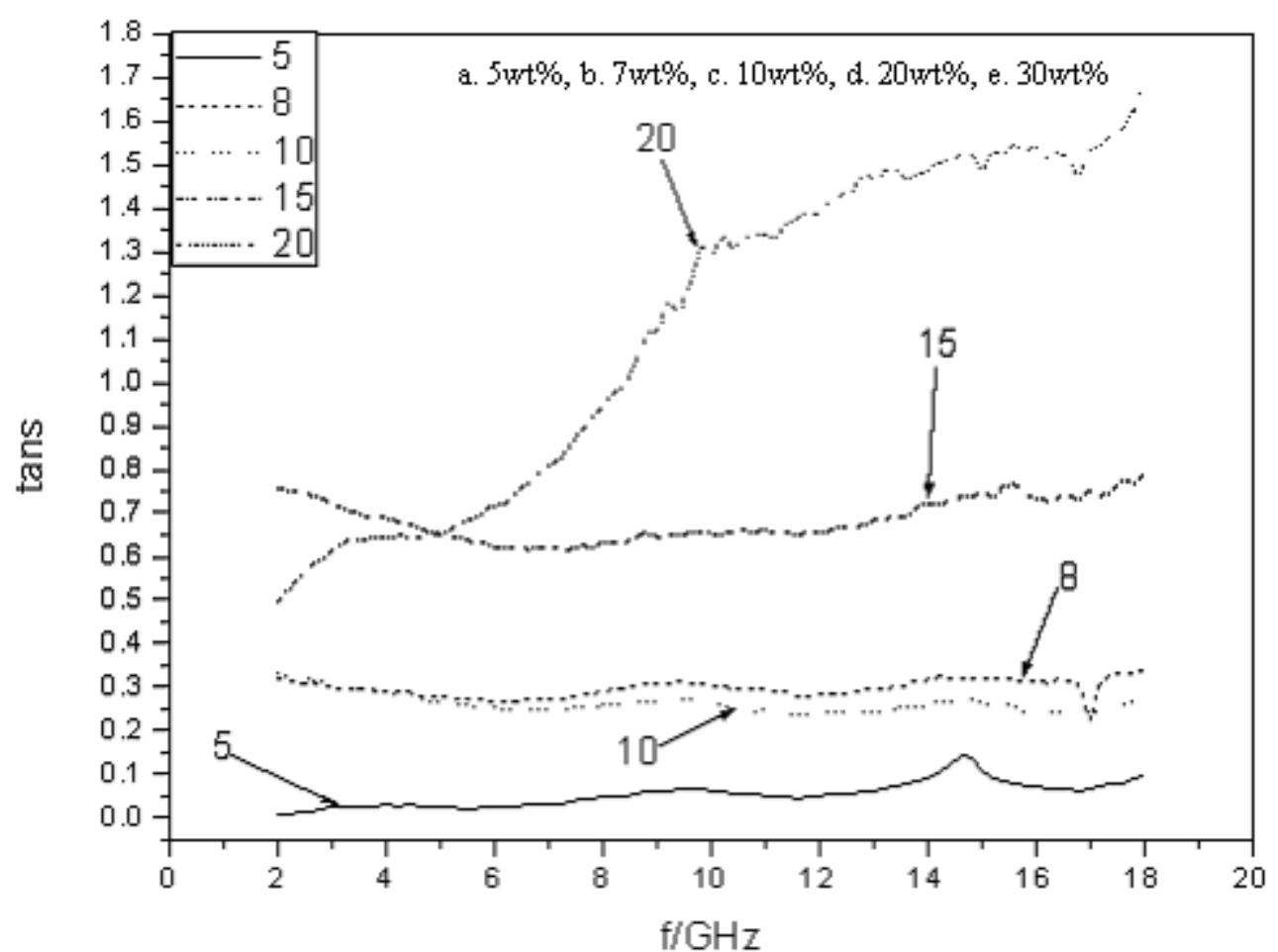

Figure 3. Relationship between $\operatorname{tg} \delta$ and frequency 


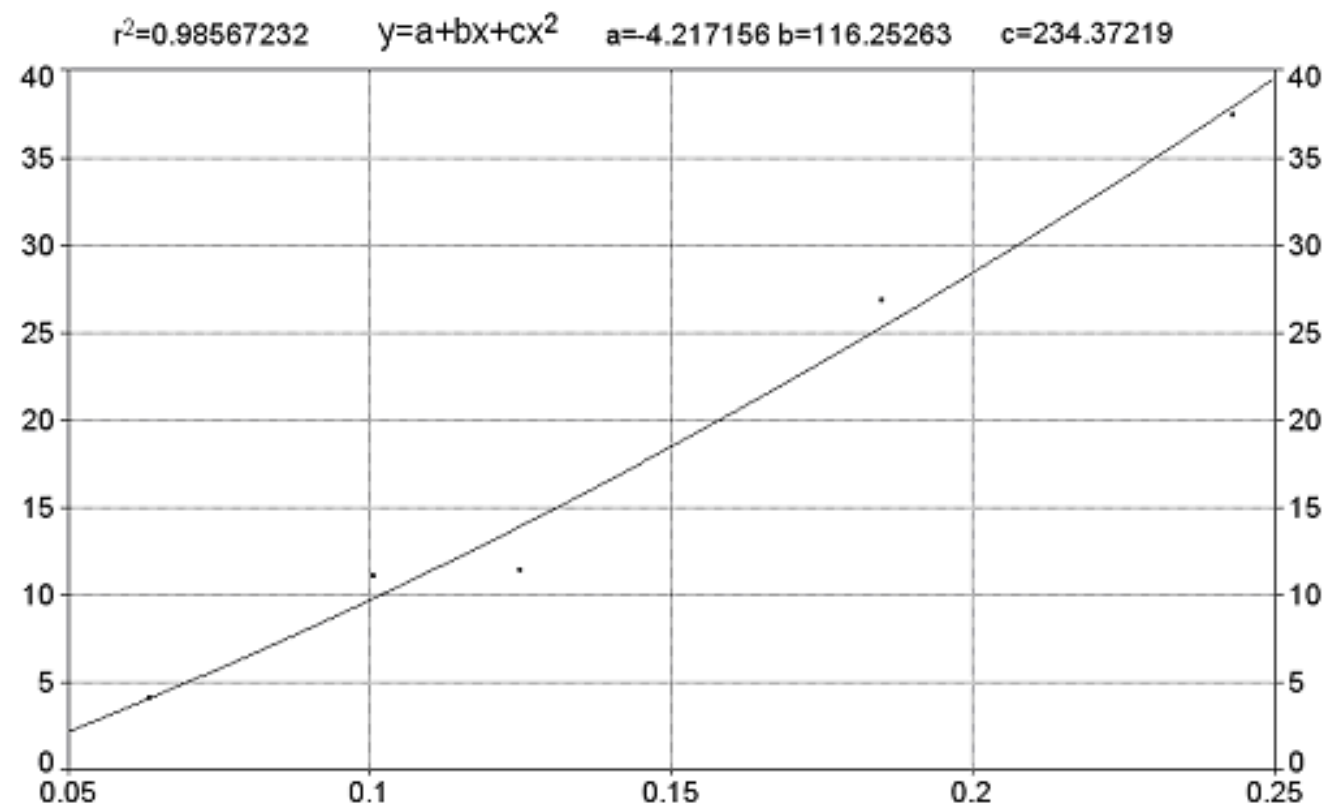

Figure 4. Relationship between $\varepsilon^{\prime}$ in $10 \mathrm{GHz}$ and nanometer power volume fraction (v)

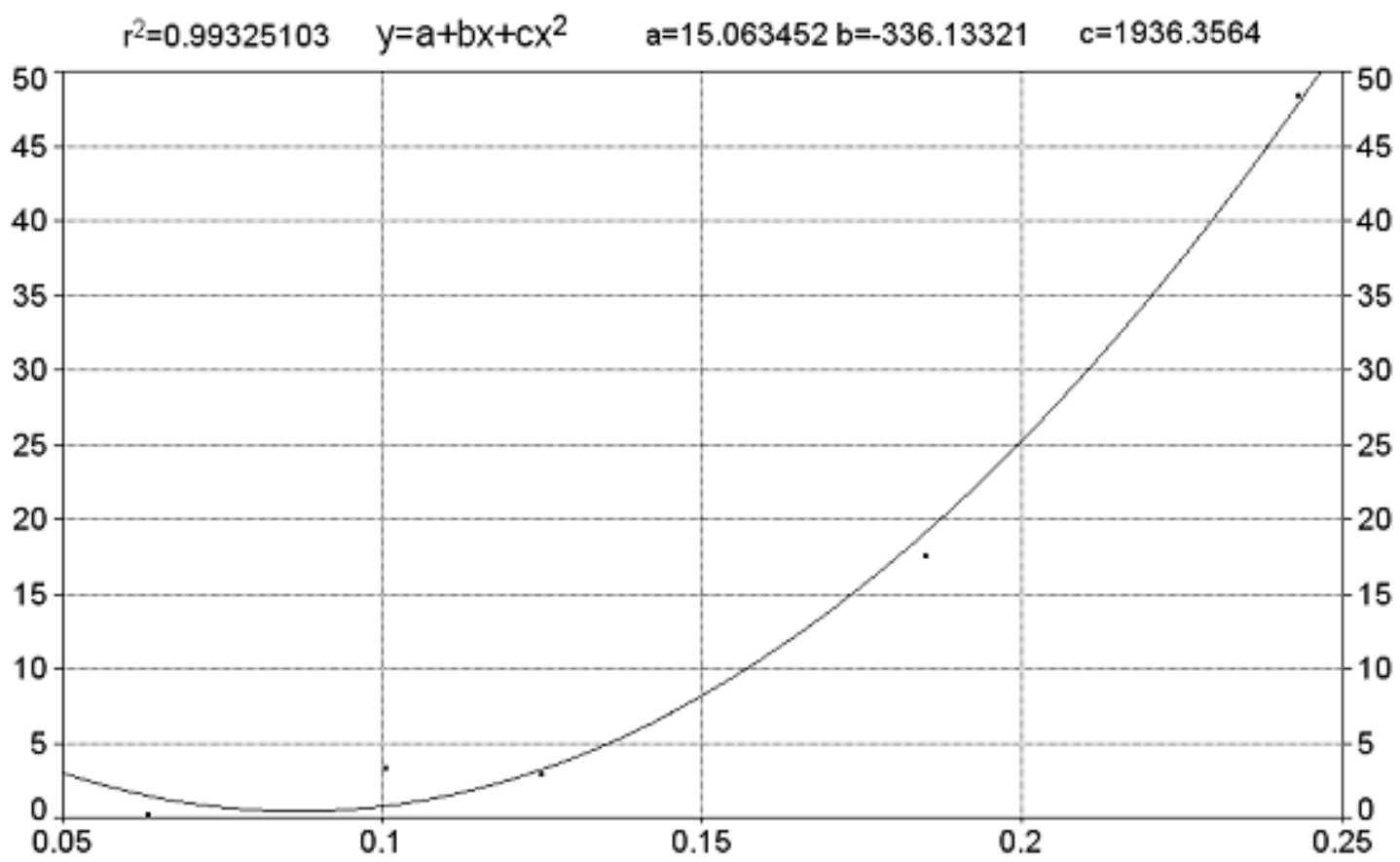

Figure 5. Relationship between $\varepsilon^{\prime \prime}$ in $10 \mathrm{GHz}$ and nanometer power volume fraction (v) 